\title{
Efficacy of a nicotine mouth spray in smoking cessation: a randomised, double-blind trial
}

\author{
Philip Tønnesen*, Hans Lauri\#, Roland Perfekt* , Karl Mann ${ }^{\top, \S}$ and Anil Batra ${ }^{+, \S}$
}

\begin{abstract}
A nicotine mouth spray has advantages over other acute forms of nicotine replacement therapy, such as a faster uptake of nicotine and faster relief of craving.
\end{abstract}

This multicentre, randomised (2:1), double-blind, placebo-controlled efficacy and safety study evaluated self-reported, carbon monoxide-verified continuous abstinence from smoking from week 2 until weeks 6, 24, and 52 in 479 smokers ( $\geqslant 1$ cigarette per day) who were treated with either active $(n=318)$ or placebo $(n=161)$ spray for 12 weeks and low-intensity counselling at three smoking cessation clinics in Denmark and Germany.

Active treatment yielded significantly higher continuous abstinence rates than placebo from week 2 until week 6 (26.1\% versus 16.1\%; relative success rate (RR) 1.62, 95\% Cl 1.09-2.41), week 24 (15.7\% versus 6.8\%; RR 2.30, 95\% Cl 1.23-4.30), and week 52 (13.8\% versus 5.6\%; RR 2.48, 95\% Cl 1.24-4.94). Most adverse events were mild to moderate, and $9.1 \%$ of subjects on active spray withdrew due to adverse events, compared to $7.5 \%$ on placebo. The overall rate of treatmentrelated adverse events was $87.4 \%$ with active spray versus $71.4 \%$ with placebo spray.

Nicotine mouth spray delivered significantly higher 6-, 24- and 52-week continuous abstinence rates than placebo.

KEYWORDS: Continuous abstinence, intention-to-treat, low-intensity counselling, nicotine replacement therapy, placebo-controlled

$\mathbf{N}$ icotine replacement therapy (NRT) is the most widely documented therapy for smoking cessation, with an overall reported success ratio (RR) of 1.5 compared with placebo [1]. It is available as several different formulations, including transdermal patch, chewing gum, sublingual tablet or lozenge, inhaler and nasal spray. The patch is a "fixed" delivery format, whereas the other formulations can be considered acute or flexible formats. As the principal action of NRT is relief of withdrawal symptoms, including craving [2], it may be possible to increase the efficacy of NRT if new formats reduce these symptoms faster and more effectively. Existing NRT formats are limited by relatively slow systemic absorption of nicotine, with the possible exception of nicotine nasal spray; however, the nasal spray causes marked initial local irritation, which restricts its appeal.

A new nicotine mouth spray (NMS), which delivers $1 \mathrm{mg}$ nicotine per spray, has been developed to enable more rapid nicotine absorption than from existing oral NRT formats. A singledose pharmacokinetic study reported a shorter time to maximum plasma nicotine concentration
(10-12.5 min post-administration), and significantly higher area under the plasma-concentration time curve during the first $10 \mathrm{~min}$, with NMS $1 \mathrm{mg}$ or $2 \mathrm{mg}$ than either $4 \mathrm{mg}$ nicotine lozenge or gum [3]. Unpublished results from a multipledose pharmacokinetic study show that comparable doses of $4 \mathrm{mg}$ nicotine per hour (NMS $2 \mathrm{mg}$ every $30 \mathrm{~min}$ ) resulted in steady-state plasma levels somewhat higher than those for gum (14$22 \%$ ) or lozenge $(10-15 \%)$. Unpublished results from a craving relief study demonstrate that two doses of NMS $1 \mathrm{mg}$ reduced craving significantly faster than either nicotine lozenge $2 \mathrm{mg}$ or $4 \mathrm{mg}$ (both unpublished studies were presented by A. Hansson, R. Perfekt and H. Kraiczi at the Society for Research on Nicotine and Tobacco 17th Annual Meeting, Toronto, ON, Canada, 2011).

The primary objective of our study was to evaluate the efficacy of NMS $1 \mathrm{mg}$ per spray versus placebo in achieving continuous abstinence from smoking from week 2 until week 6, 24 and 52, respectively. In an effort to replicate an over-the-counter situation, counselling was as unobtrusive as possible.
AFFILIATIONS

*Dept of Pulmonary Medicine, Gentofte University Hospital, Copenhagen, Denmark.

\#Global Medical Affairs and Clinical Research, McNeil AB, Helsingborg, Sweden.

-Dept of Addictive Behaviour and Addiction Medicine, Central Institute of Mental Health, University of Heidelberg,

${ }^{+}$Dept of Psychiatry and

Psychotherapy, Section for Addiction Medicine and Research, University Hospital of Tübingen, Germany.

${ }^{5}$ Both authors contributed equally.

CORRESPONDENCE

P. Tønnesen

Dept of Pulmonary Medicine

Gentofte University Hospital DK-2900 Hellerup

Denmark

E-mail: philipt@dadlnet.dk

Received:

Sept 082011

Accepted after revision:

Jan 112012

First published online:

Feb 092012

This article was modified in April 2016 to correct errors in the licence information.

European Respiratory Journal Print ISSN 0903-1936 Online ISSN 1399-3003 


\section{MATERIAL AND METHODS}

\section{Study design and conduct}

This randomised, double-blind, placebo-controlled, parallelgroup, 52-week study was performed at three smoking cessation clinics in Denmark (Copenhagen) and Germany (Mannheim and Tübingen) between March 2009 and June 2010. Daily cigarette smokers were recruited through advertisements in local newspapers. Following telephone screening, potential subjects attended the clinic for baseline assessments. Admission criteria are shown in table 1 .

Eligible subjects were randomised to receive either NMS or placebo spray (ratio 2:1) stratified by study site. Subjects were instructed to quit smoking completely the day after the baseline visit and to start using the spray. The study comprised 11 clinic visits (baseline, and week 1, 2, 4, 6, 8, 12, 16, 20, 24 and 52) and four telephone contacts between weeks 24 and 52 . Low-intensity counselling was provided, with general written and brief $(<10 \mathrm{~min})$ verbal smoking cessation advice at baseline, and very brief $(<3 \mathrm{~min})$ verbal cessation advice at subsequent visits up to and including week 24 . The primary study end-point was self-reported, objectively verified continuous abstinence from smoking from week 2 until weeks 6, 24 and 52. The study protocol was approved by local independent ethics committees and all subjects provided verbal and written, signed, informed consent before entering the study.

\section{Study treatment}

All study medications were manufactured by McNeil AB, Helsingborg, Sweden. The NMS (1 mg of nicotine per spray after priming) and placebo spray contained 150 metered spray doses for administration into the mouth. The nicotine solution is clear to weakly opalescent, colourless to light yellow, with a peppermint scent. The placebo was identical in appearance, but contained capsaicin instead of nicotine to mimic the taste of nicotine.

\section{Dosage instructions}

During weeks 1-6, subjects were instructed to use one to two sprays when they normally would have smoked a cigarette or when they experienced an urge to smoke; the second spray could be used if cravings were not reduced within a few minutes of the first spray. For many smokers, this meant one to two sprays every 30-60 min; the recommended maximum dose was four sprays per hour, and 64 sprays per day. After the 6-week full dose period, subjects were instructed to reduce spray use so that by the end of week 9 they were using half of the average number of sprays used per day during weeks 1-6, then to continue to reduce to not more than four sprays per day by week 12 . Occasional use (not more than four sprays per day) was permitted during weeks 13-24.

\section{Assessments}

Smoking status was assessed at all visits, using self-reports, objectively confirmed by measuring carbon monoxide (CO) levels in exhaled air after a 15-s breath hold using a CO monitor (Micro CO Monitor, Cardinal Health, Basingstoke, UK). A CO level $\geqslant 10 \mathrm{ppm}$ classified the subject as a smoker.

During the first 4 weeks, subjects self-reported daily use of study drug, adverse events and craving and withdrawal symptoms experienced during the previous $24 \mathrm{~h}$, using a Palm Treo ${ }^{\mathrm{TM}} 650$ portable electronic diary (eDiary; software by CRF Health, Helsinki, Finland). From weeks 4 to 12 only daily use of spray and adverse events were recorded in the eDiary.

In addition, craving and withdrawal symptoms (desire/urge to smoke, irritability/frustration/anger, restlessness, difficulty concentrating, anxiety, dysphoric or depressed mood, insomnia

\section{TABLE 1 Inclusion and exclusion criteria}

Inclusion criteria

Males and females, 18 yrs or older

Daily cigarette smoker for the last 3 yrs or more (no lower limit in number of daily cigarettes)

Expired carbon monoxide level of at least $10 \mathrm{ppm}$ after at least 15 smoke-free min

Motivated and willing to completely stop smoking from the day after the baseline visit and to stay smoke-free

Willing to use study drug for at least 12 weeks

Female participants of child-bearing potential to use a medically acceptable method of birth control

Evidence of a personally signed and dated informed consent document

Willing and able to comply with all study procedures and attend 11 scheduled visits during the 52-week study

Willing and able to use an eDiary, according to the instructions provided, and willing to return the eDiary at the 12 -week visit
Exclusion criteria

Current use of tobacco-containing products, other than cigarettes, or smoking other substances

Use of other NRT, or bupropion or varenicline, or any other treatment for tobacco dependence (e.g. acupuncture)

Unstable angina pectoris or myocardial infarction or stroke during the previous 3 months

Pregnancy, lactation or intended pregnancy

Suspected alcohol or drug abuse

Another member of the same household who is already a subject in the current trial

Participation in any other clinical trial within the previous 3 months, or during the current trial

Any acute or chronic medical or psychiatric condition or previously diagnosed renal or hepatic disease that may increase the risk associated with study participation

Presence of an oral lesion (suspected malignant lesion and/or erosive lesion) that required further investigation, such as biopsy 
and increased appetite [4]) were rated on a 5-point scale $(0=$ not at all, $1=$ somewhat, $2=$ moderate, $3=$ very much, $4=$ extreme) at all visits from week 4 to week 24 .

Safety data were collected using open-ended questions at all visits and was also captured daily in the eDiary during weeks 1-12 using a checklist of 14 predefined adverse events (table 2). A visual mouth inspection was performed by site dentists at baseline and at weeks 2, 12 and 24. Treatment acceptability was assessed at weeks 1, 6 and 12. Blood pressure and pulse were assessed at every visit from baseline to week 24 and a physical examination (heart, lungs and visible findings) performed at baseline and week 24 . Weight was measured at baseline and at weeks $6,12,16,20,24$ and 52 .

Saliva samples for cotinine analysis were taken at baseline and weeks 2, 6, 12 and 24, to determine nicotine substitution levels. Pregnancy tests were performed at baseline and regularly up to week 24 .

\section{Statistics}

The sample size determination was based on an assumed continuous abstinence rate of $40 \%$ in the active group at week 6. The study was powered to detect an odds ratio for success of at $\geqslant 2$ versus placebo at week 6 with a probability of $90 \%$. The unbalanced randomisation ratio (active:placebo 2:1) was chosen in order to get a sufficiently large safety database for the active treatment. Under these premises, a Chi-squared test with significance level 5\% required 307 subjects in the NMS group, and 154 in the placebo group.
Allocation to treatment group was based on a subject randomisation list stratified by study site. The supply or resupply of study medication to a subject was determined via an interactive voice response system involving a dispenser pack number randomisation list. Both randomisation lists were computer-generated and were devised by the Biometrics and Clinical Data Systems Department, McNeil-PPC, Inc., Fort Washington, PA, USA.

Continuous abstinence was defined as self-reported "no smoking", verified by a $\mathrm{CO}$ value $<10 \mathrm{ppm}$, from week 2 up to and including the given visit. Any subject who missed the visit(s) at week(s) 8,16 and/or 20, or for some other reason had missing $\mathrm{CO}$ value(s) at one or more of these visits, was not regarded a treatment failure if the subject was verified continuously abstinent at a later visit. Point prevalence abstinence at a given visit was defined as $\mathrm{CO}$-verified, self-reported non-smoking for the previous 7 days or longer.

Continuous and 7-day point prevalence abstinence rates for the two treatment groups were compared for each visit from week 4 onwards using Pearson's Chi-squared test. Point estimates and $95 \%$ CI were calculated for the relative success rates (RR) and for the odds ratio for success with active versus placebo. All significance tests were two-sided and performed at the $5 \%$ level. All randomised subjects received study medication and were included in both the full (intention-to-treat) and safety analysis sets. Analysis of the primary end-points was performed in a hierarchical setting ensuring an overall, joint significance level of $5 \%$. All confidence intervals were calculated by using normal approximations. The Mann-Whitney U-test was used to

TABLE 2 Treatment-related adverse events: overview and numbers of subjects reporting (by preferred term)

\begin{tabular}{|c|c|c|}
\hline & Nicotine mouth spray & Placebo \\
\hline Subjects $\mathrm{n}$ & 318 & 161 \\
\hline Subjects with at least one adverse event ${ }^{\#}$ & $278(87.4)$ & $115(71.4)$ \\
\hline Subjects discontinued because of an adverse event & $25(7.9)$ & $11(6.8)$ \\
\hline \multicolumn{3}{|c|}{$\begin{array}{l}\text { Adverse events (preferred term) reported by } \geqslant 10 \% \text { of subjects in either } \\
\text { treatment group }\end{array}$} \\
\hline Headache & $103(32.4)$ & $58(36.0)$ \\
\hline Nausea & $101(31.8)$ & $39(24.2)$ \\
\hline Dyspepsia & $93(29.2)$ & $24(14.9)$ \\
\hline Stomatitis & $75(23.6)$ & $27(16.8)$ \\
\hline Salivary hypersecretion & $68(21.4)$ & $19(11.8)$ \\
\hline Dizziness & $61(19.2)$ & $39(24.2)$ \\
\hline Burning sensation in mouth & $38(11.9)$ & $13(8.1)$ \\
\hline
\end{tabular}

Data are presented as $\mathrm{n}(\%)$, unless otherwise stated. ${ }^{*}$ : adverse events reported by number of subjects in either treatment group who reported a treatment-related adverse event at least once. "Treatment related" was captured in the case report forms as the answer "Yes/Unknown" to the question "Is there a reasonable possibility the adverse event is related to study treatment?" All the preferred terms in the table above were included as a "health problem" prompt in the eDiary. The "health problem" prompt consisted of a checklist of 14 predefined adverse events (the 12 listed above, plus common cold and vomiting) and an "Other" option. The preferred terms dyspepsia, stomatitis, salivary hypersecretion, dysgeusia, and burning sensation in the mouth were elicited using the following adverse event prompts in the eDiary: heartburn, mouth irritation, excessive salivation, altered taste, and burning lips, respectively. 
compare treatments with respect to craving and withdrawal symptom scores.

\section{RESULTS}

\section{Subject characteristics}

A total of 479 smokers were enrolled in the study between March and June 2009; 318 received active treatment and 161 placebo. A total of 242 subjects completed the study (fig. 1).

The subjects' demographic characteristics were similar in both treatment groups (table 3).

\section{Smoking cessation}

Compared to placebo, rates of CO-verified, continuous abstinence from week 2 were statistically significantly higher with NMS at all three primary assessment time points, i.e. at weeks 6, 24 and 52 (table 4). Most relapses occurred early in the study, and continuous abstinence rates stabilised during the 6 months from weeks 24-52.

The superiority of NMS was further supported by comparisons of both continuous abstinence rates at intermediate visits

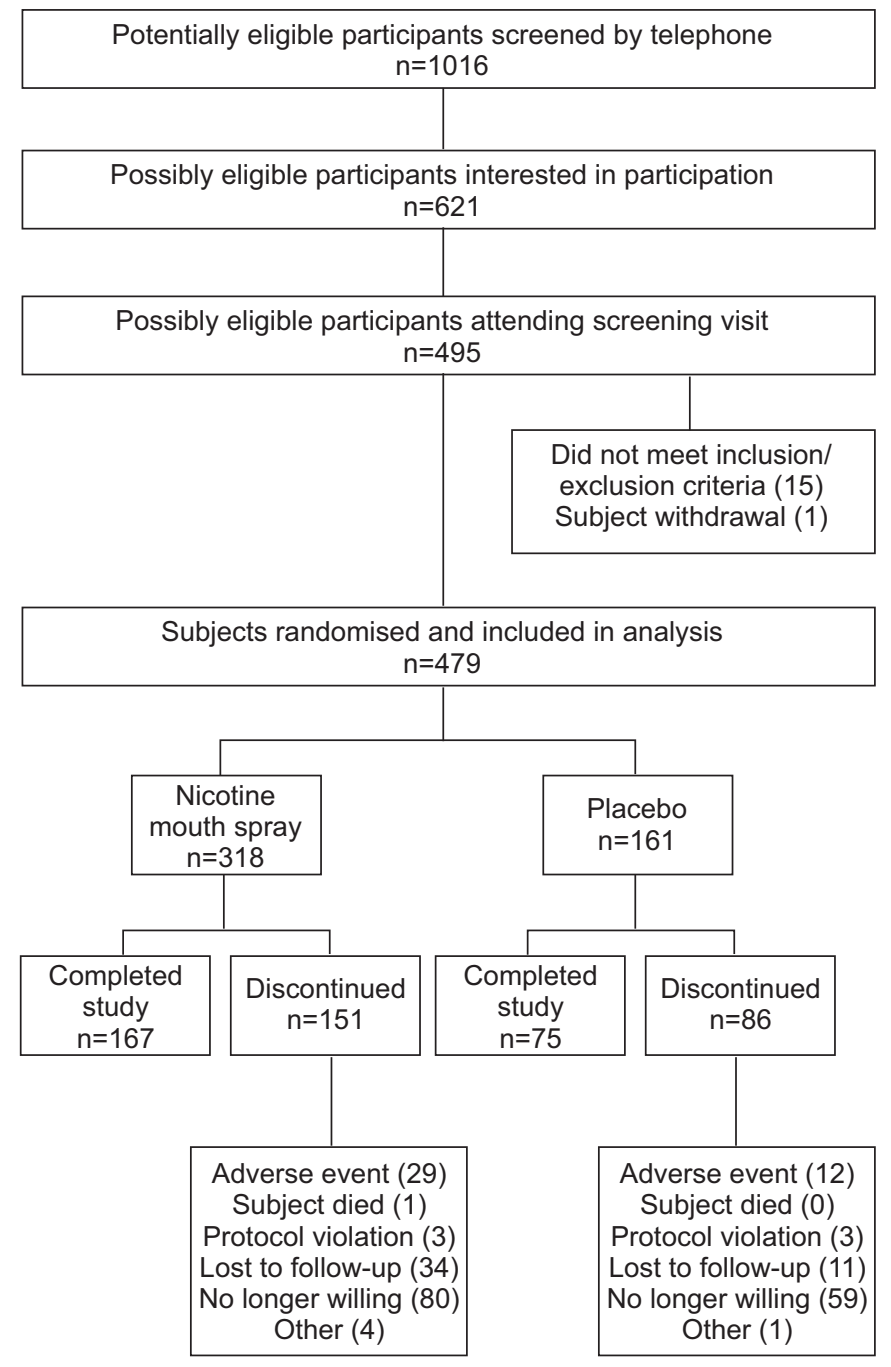

FIGURE 1. Flow diagram of the progress of subjects through the phases of the study. (table 4) and 7-day point prevalence abstinence rates (fig. 2) from week 4 onwards. The $\mathrm{CO}$-verified point prevalence abstinence rates were statistically significantly higher with NMS at all assessment time points; the week 52 comparison was $21.4 \%$ (NMS) versus $8.1 \%$ (placebo) (RR 2.65, 95\% CI 1.51-4.65).

\section{Spray use}

The median of the individual mean number of active sprays used per day among subjects with eDiary reports was 13.1 (interquartile range (IQR) 7.5-20.7), during the first week, which declined to 10.2 (IQR 2.9-17.8) and 4.3 (IQR 0-9.8) in weeks 6 and 12 , respectively, with a wide inter-individual range. Higher spray use was observed in the placebo group during the first 6 weeks, probably due to lack of nicotine (fig. 3).

Subjects with verified 7-day point prevalence abstinence on NMS exhibited a steep rate of decrease in median saliva cotinine levels. Median cotinine values relative to baseline dropped from 37\% (IQR 17-69\%) at week $2(n=115)$ to $19 \%$ (IQR $4-48 \%)$ at week $6(n=99)$. Only $37 \%$ and $24 \%$ of these subjects had substitution levels above $50 \%$ at week 2 and week 6 , respectively.

\section{Craving symptoms}

The means of the individual weekly average craving scores of abstinent subjects using NMS were lower than those using placebo throughout (mean scale unit difference 0.3-0.4); the inter-group differences were statistically significant for weeks 1 and 2, but not for weeks 3 and 4 .

\section{Weight}

At week 24, median weight increases in subjects verified as continuously abstinent from week 2 onwards were $4.9 \mathrm{~kg}$ $(\mathrm{n}=50$; range $-5.0-15.1 \mathrm{~kg})$ with NMS and $4.2 \mathrm{~kg}(\mathrm{n}=11$; range $-1.6-17.3 \mathrm{~kg}$ ) with placebo ( $p=0.93$, Mann-Whitney U-test).

\section{Spray acceptability}

At week 1,52.5\% of reporting subjects on active treatment rated the overall spray acceptability to be 8,9 or $10(1=$ very poor, $10=$ excellent), $59.5 \%$ rated the NMS either "very" or "extremely effective" in dealing with cravings, and 51.3\% rated the speed of action either "very" or "extremely" fast.

\section{Safety}

A total of 425 subjects $(91.8 \%$ of NMS and $82.6 \%$ of placebo subjects) reported 2,673 adverse events (1,927 with NMS, 746 with placebo), and 393 subjects (87.4\% of NMS and $71.4 \%$ of placebo subjects) reported 2,044 treatment-related adverse events (1,504 with NMS, 540 with placebo (table 2)). Eight of the 12 most common treatment-related adverse events (hiccups, throat irritation, nausea, dyspepsia, mouth irritation, salivary hypersecretion, burning sensation in mouth, and constipation) were more prevalent with NMS (table 2). Of the treatment-related adverse events reported with NMS, $61.8 \%$ were mild, $26.6 \%$ were moderate and $6.3 \%$ were severe (severity was missing for $5.4 \%$ ).

24 subjects reported serious adverse events (SAEs), 16 using NMS and eight using placebo. None of the 16 SAEs in NMS users (cancer (breast and lung), back pain, foreign body in lungs, fracture (ankle and femur), breast cyst, herpes simplex infection, 
TABLE 3 Baseline demographic characteristics and smoking history

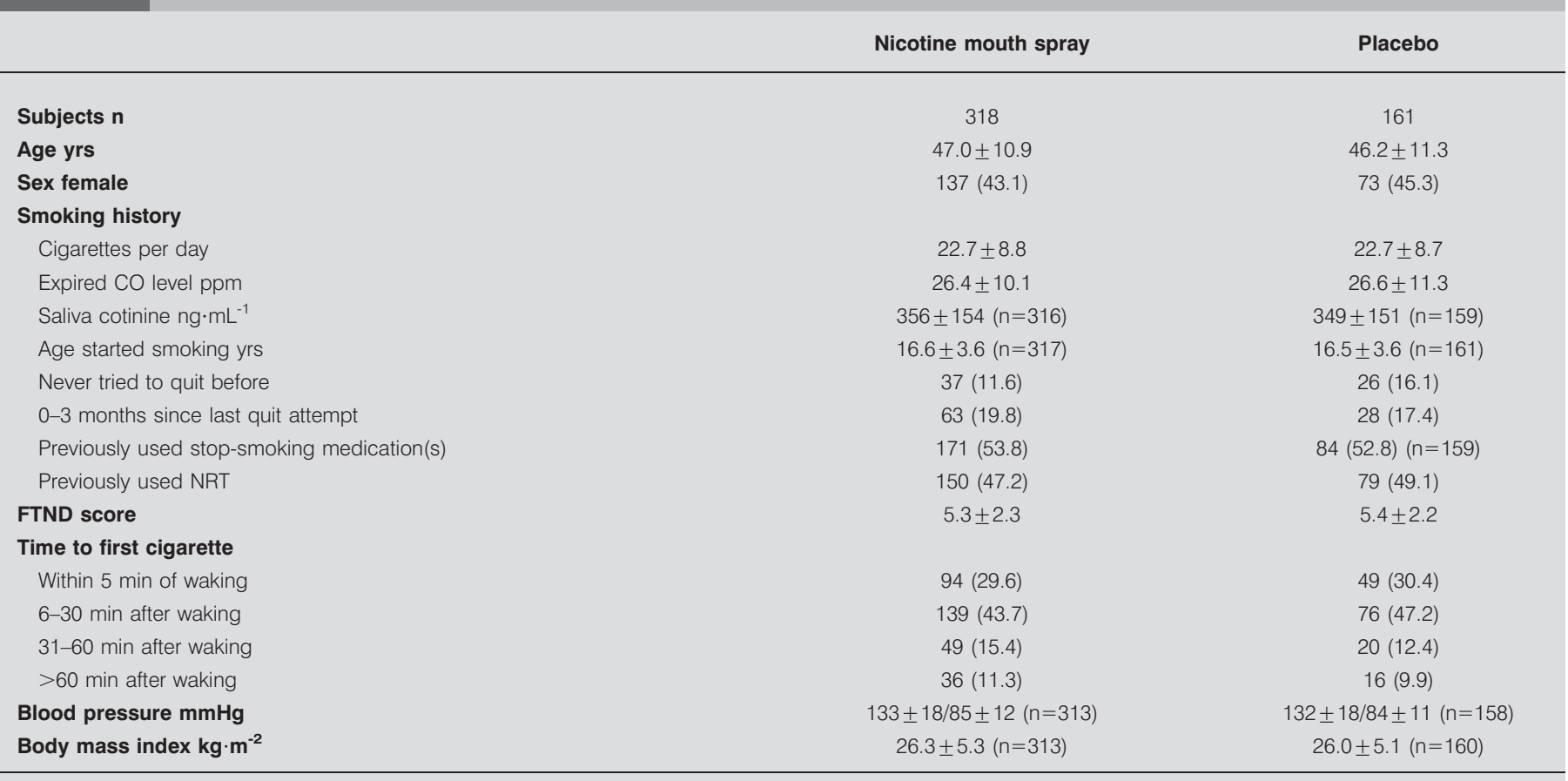

Data are presented as mean \pm SD or $n(\%)$, unless otherwise stated. CO: carbon monoxide; NRT: nicotine replacement therapy; FTND: Fagerström Test of Nicotine Dependence.

contusion, rupture of shoulder tendons, goitre, neck pain, gastric bleeding, shortness of breath, myocardial infarction and death) were considered related to study treatment.

The subject who died was a 47-yr-old male who had smoked 10 cigarettes per day for 33 yrs. He used approximately 11 sprays per day and had stopped smoking. After 11 days he was found dead and the cause of death was not clarified at autopsy. The venous blood nicotine concentration was $7 \mathrm{ng} \cdot \mathrm{mL}^{-1}$. The acute myocardial infarction (AMI) occurred in a 58-yr-old male who had smoked 27 cigarettes per day for 40 yrs. He used approximately 10 sprays of NMS per day before permanently stopping treatment 4 days prior to the AMI (6 months into the study). However, he was still smoking 10 cigarettes per day. He suffered from peripheral arterial disorder in the legs and coronary artery stenosis and had stents implanted.

Nine SAEs occurred in eight subjects in the placebo group.

\section{Visual mouth inspection}

No subjects had worsened oral abnormalities or lesions during the study. New oral abnormalities or lesions were reported for 18 subjects $(5.7 \%)$ using NMS and seven subjects (4.3\%) using placebo. The incidence of abnormalities and lesions was very low $(<2 \%)$ for all events and was similar between treatment groups. Most events were rated as mild; none were severe.

TABLE 4 Carbon monoxide-verified continuous abstinence rates from week 2 to weeks 4, 6, 8, 12, 16, 20, 24 and 52

\begin{tabular}{|c|c|c|c|c|c|}
\hline Time point & Nicotine mouth spray\# & Placebo & p-value ${ }^{+}$ & Risk ratio $(95 \% \mathrm{CI})^{\S}$ & Odds ratio $(95 \% \mathrm{Cl})^{\S}$ \\
\hline Week 4 & $101(31.8)$ & $35(21.7)$ & 0.022 & $1.46(1.05-2.04)$ & $1.68(1.08-2.61)$ \\
\hline Week 8 & $78(24.5)$ & $23(14.3)$ & 0.009 & $1.72(1.12-2.63)$ & $1.95(1.17-3.25)$ \\
\hline Week12 & $64(20.1)$ & $21(13.0)$ & 0.055 & $1.54(0.98-2.43)$ & $1.68(0.98-2.87)$ \\
\hline Week 16 & $57(17.9)$ & $15(9.3)$ & 0.013 & $1.92(1.13-3.29)$ & $2.13(1.16-3.89)$ \\
\hline Week 52 & $44(13.8)$ & $9(5.6)$ & 0.007 & $2.48(1.24-4.94)$ & $2.71(1.29-5.71)$ \\
\hline
\end{tabular}

Values are presented as $n(\%)$, unless otherwise stated. ${ }^{\#}: n=318 ;{ }^{\bullet}: n=161 ;{ }^{+}: p$-values were calculated using the Chi-squared test; ${ }^{5}$ : estimated risk ratios/odds ratios and corresponding $95 \%$ confidence intervals were calculated using Mantel-Haenszel statistics. 


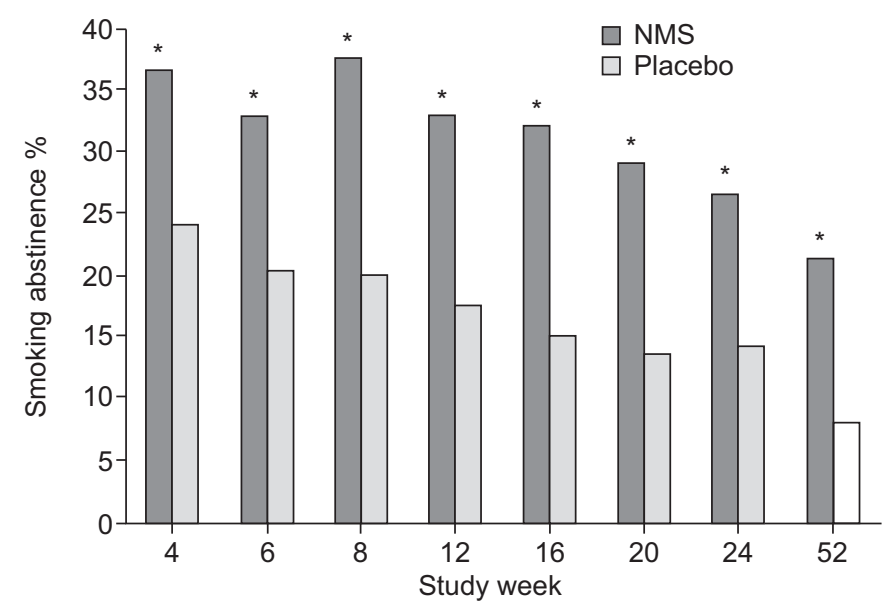

FIGURE 2. Proportion of subjects with carbon monoxide-verified 7-day point prevalence of abstinence. Includes all subjects (nicotine mouth spray (NMS) $n=318$; placebo: $n=161) .{ }^{*}: p<0.05$ between the abstinence rates in the NMS group compared with the placebo group.

\section{DISCUSSION}

This study demonstrated the efficacy and safety of NMS $1 \mathrm{mg}$ for smoking cessation. Active spray was superior to placebo with respect to abstinence up to 6, 24 and 52 weeks, with $53 \%$ of subjects continuously abstinent at week 6 still abstinent at week 52. The observed RR of 2.48 at $1 \mathrm{yr}$ is higher than that for other NRT formulations. For NRT the relative efficacy has been fairly consistent across the level of support and different settings, such as over-the-counter, general physician practices and specialist smoking cessation services [5]. However, our 1 -yr absolute abstinence rate of $13.8 \%$ was lower than that in several previous smoking cessation studies [6], probably due to the minimal counselling employed in our study. It has been shown that quit rates increase with increasing intensity of counselling and number of clinic visits [7].

The median nicotine substitution level after 2 weeks among point prevalence abstinent subjects on active treatment was low. This was reflected by $65-85 \%$ of abstinent subjects in the active group having average daily doses of spray regularly below their baseline number of cigarettes per day, during the 6-week full treatment period. Two trials that addressed scheduling of NRT as a way of improving adherence compared a fixed-dose regimen of nicotine $2 \mathrm{mg}$ gum against a smoking-urge-driven regimen $[8,9]$. The fixed-dose regimen had higher quit rates (and higher treatment usage) but the difference was nonsignificant (RR 1.22, 95\% CI 0.92-1.61) [1]. Our study employed a new, more intuitive, schedule that essentially combined an urge-driven and a fixed-dose regimen, i.e. a flexible schedule (subjects were asked to take a spray when they would normally have smoked a cigarette, or experienced cravings) that also included a typical fixed schedule for most smokers. One option for the future with the aim of increasing the overall level of nicotine substitution could be to use a combination of NMS plus nicotine patch, which delivers a more constant level of nicotine [7]. Combination therapy with two types of NRT has been shown to increase quit rates compared to monotherapy [6].

Despite the low nicotine substitution levels attained with NMS, use of active spray resulted in higher observed efficacy relative

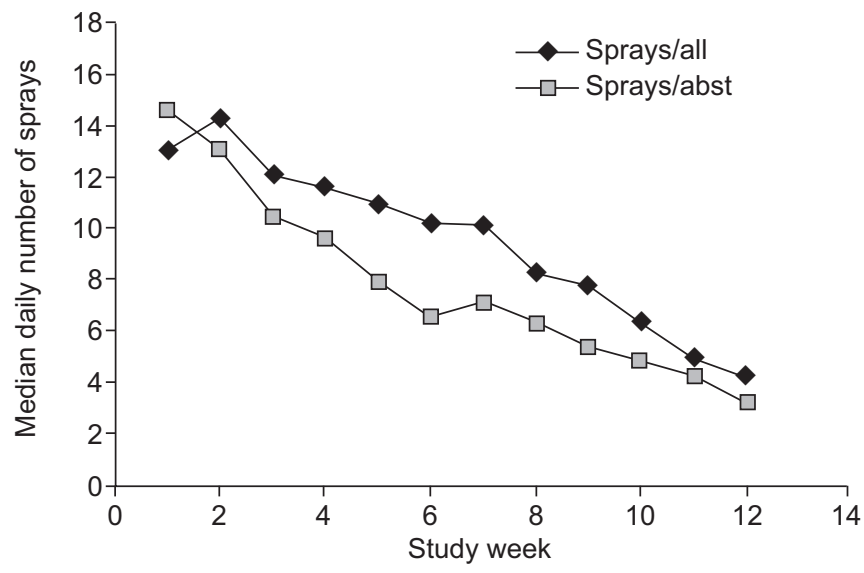

FIGURE 3. Median of the individual mean daily number of spray doses by study week (weeks 1 to 12) in subjects in the nicotine mouth spray (NMS) group. Sprays/all: all NMS subjects reporting data for at least $50 \%$ of the days in that specific period; Sprays/abst: all NMS subjects with verified 7-day point prevalence abstinence reporting data for at least $50 \%$ of the days in that specific period.

to placebo. This could possibly be attributed to the more rapid absorption of nicotine compared to other oral NRTs and thereby faster relief of cravings (unpublished results presented by A. Hansson, R. Perfekt and H. Kraiczi at the Society for Research on Nicotine and Tobacco 17th Annual Meeting). This explanation is supported by the lower mean craving scores among abstinent subjects using active NMS compared to placebo during the first few weeks. A relationship has been reported between the severity of some withdrawal symptoms, including craving, and outcome of attempts to quit smoking [10-12].

Although almost $90 \%$ of subjects using active spray reported adverse events, the number of participants who withdrew due to adverse events was low (NMS 9.1\%, placebo 7.5\%), and was comparable to other NRTs or varenicline in smoking cessation studies [13-17]. The high incidence of hiccups with NMS was probably secondary to irritation from swallowed nicotine, so current patient instructions advise users not to swallow the spray straight after spraying.

In summary, NMS is effective in helping smokers quit and, with a relative success rate of 2.48 compared to placebo regarding abstinence at $1 \mathrm{yr}$, might have greater relative efficacy than other forms of NRT. The NMS seems to be well tolerated during use, with an expected adverse event profile.

\section{CLINICAL TRIAL}

This study is registered at ClinicalTrials.gov with identifier number NCT00882375.

\section{STATEMENT OF INTEREST}

Statements of interest for all authors of this manuscript and for the study itself can be found at www.erj.ersjournals.com/site/misc/ statements.xhtml

\section{ACKNOWLEDGEMENTS}

We thank the other study investigators (Z. Saghir and H. Ashraf, Gentofte Hospital, Copenhagen; P. Holmstrup, Dental University School, Copenhagen, Denmark; M. Fischer, H. Friederich and T-H. Waidelich, University of Heidelberg, Heidelberg; J. El-Kasmi, C. von Ohle, A. Diehl, K. Isaac and H. Krämer, University Hospital, Tubingen, Germany), the 
study team at McNeil AB, Helsingborg, Sweden, and Anne Hendrie (medical writer, Shropshire, UK) who edited the final paper.

\section{REFERENCES}

1 Stead LF, Perera R, Bullen C, et al. Nicotine replacement therapy for smoking cessation. Cochrane Database Syst Rev 2008; 1: CD000146.

2 Benowitz NL. Clinical pharmacology of nicotine: implications for understanding, preventing, and treating tobacco addiction. Clin Pharmacol Ther 2008; 83: 531-541.

3 Kraiczi H, Hansson A, Perfekt R. Single-dose pharmacokinetics of nicotine when given with a novel mouth spray for nicotine replacement therapy. Nicotine Tob Res 2011; 13: 1176-1182.

4 American Psychiatric Association (APA). Diagnostic and Statistical Manual of Mental Disorders. 4th Edn Text Revision (DSM-IV-TR). Washington, APA, 2000.

5 Tønnesen P, Paoletti P, Gustavsson G, et al. Higher dosage nicotine patches increase one-year smoking cessation rates: results from the European CEASE trial. Eur Respir J 1999; 13: 238-246.

6 Tønnesen P. Smoking cessation: how compelling is the evidence? A review. Health Policy 2009; 91: S15-S25.

7 Fiore MC, Jaén CR, Baker TB, et al. Treating Tobacco Use and Dependence: 2008 Update. Clinical Practice Guideline. Rockville, US Department of Health and Human Services, Public Health Service, 2008.

8 Goldstein MG, Niaura R, Follick MJ, et al. Effects of behavioral skills training and schedule of nicotine gum administration on smoking cessation. Am J Psychiatry 1989; 146: 56-60.
9 Killen JD, Fortmann SP, Newman B, et al. Evaluation of a treatment approach combining nicotine gum with self-guided behavioral treatments for smoking relapse prevention. J Consul Clin Psychol 1990; 58: 85-92.

10 Doherty K, Kinnunen T, Militello FS, et al. Urges to smoke during the first month of abstinence: relationship to relapse and predictors. Psychopharmacology 1995; 119: 171-178.

11 Killen JD, Fortmann SP. Craving is associated with smoking relapse: findings from three prospective studies. Exp Clin Psychopharmacol 1997; 5: 137-142.

12 West RJ, Hajek P, Belcher M. Severity of withdrawal symptoms as a predictor of outcome of an attempt to quit smoking. Psychol Med 1989; 19: 981-985.

13 Hays JT, Ebbert JO. Adverse effects and tolerability of medications for the treatment of tobacco use and dependence. Drugs 2010; 70 : 2357-2372.

14 Ossip DJ, Abrams SM, Mahoney MC, et al. Adverse effects with use of nicotine replacement therapy among quitline clients. Nicotine Tob Res 2009; 11: 408-417.

15 Gonzales D, Rennard SI, Nides M, et al. Varenicline, an $\alpha 4 \beta 2$ nicotinic acetylcholine receptor partial agonist, vs sustainedrelease bupropion and placebo for smoking cessation: a randomized controlled trial. JAMA 2006; 296: 47-55.

16 Jorenby DE, Hays JT, Rigotti NA, et al. Efficacy of varenicline, an $\alpha 4 \beta 2$ nicotinic acetylcholine receptor partial agonist, vs placebo or sustained-release bupropion for smoking cessation: a randomized controlled trial. JAMA 2006; 296: 56-63.

17 Tonstad S, Tønnesen P, Hajek P, et al. Effect of maintenance therapy with varenicline on smoking cessation: a randomized controlled trial. JAMA 2006; 296: 64-71. 